\title{
Descriptive study of outcome of antibiotic cement-impregnated intramedullary nail in treatment of infected non-union of weight bearing long bones
}

\author{
Abhijit Sen, Chandan Kulkarni*, Ashok Meena
}

Department of Orthopaedics, SMS Medical College, Jaipur, Rajasthan, India

Received: 14 May 2019

Revised: 18 August 2019

Accepted: 29 August 2019

\author{
*Correspondence: \\ Dr. Chandan Kulkarni, \\ E-mail: cuk146@gmail.com
}

Copyright: () the author(s), publisher and licensee Medip Academy. This is an open-access article distributed under the terms of the Creative Commons Attribution Non-Commercial License, which permits unrestricted non-commercial use, distribution, and reproduction in any medium, provided the original work is properly cited.

\begin{abstract}
Background: Infected non-union of tibia and femur is a debilitating disorder for patient as well as challenging task for treating surgeon. Conventionally treatment of infected non-union is a two staged procedure. But antibiotic cementimpregnated intramedullary nailing (ACIINs) is a single staged and cost-effective procedure. Hence we intended to study the outcome of ACIIN use in infected non-union of tibia and femur.

Methods: This is a hospital based prospective case series type of descriptive study conducted in Department of Orthopedics, SMS Medical College and Hospital, Jaipur. We studied 35 cases of infected non-union of femur and tibia fracture with interlock nail in situ. All patients were treated with interlock nail removal, debridement and freshening of sclerosed bony ends and fixation with ACIIN. All were followed for at least 6 months for infection control and bony union and final results were evaluated by Paley's bony criteria and functional criteria.

Results: Infection was controlled in $94.28 \%$ cases. Bony union was achieved in $88.57 \%$ cases (19 femur and 12 tibia). Average duration for bony union was 7.3 months for femur and 8 months for tibia. According to Paley's criteria for bony outcome and functional outcome $65.71 \%$ and $51.43 \%$ had shown excellent outcome respectively.

Conclusions: ACIIN is a good modality for treatment of infected non union of tibia and femur in terms of infection control and bony union and has a good functional outcome when bone gap is less.
\end{abstract}

Keywords: Infected nonunion, Tibia, Femur, ACIIN

\section{INTRODUCTION}

Long bone fractures are severe injuries commonly resulting from high-energy trauma, usually due to roadtraffic collisions. Intramedullary (IM) nailing is considered the gold standard of treatment of closed and many open femoral and tibial shaft fractures due to its biomechanical and biological advantages. ${ }^{1}$ With the increasing number of road traffic accidents and increasing use of orthopedic devices to fix fractures, 4 to $7 \%$ of intramedullary nail get infected when it is used in compound fractures, whereas in case of closed fractures the risk remains similar to the general risk of infection after any orthopedic trauma procedure. ${ }^{1,2}$ Infected nonunion of tibia and femur is a debilitating disorder for patient as well as challenging task for treating surgeon. ${ }^{2,3}$ Most of the infections are caused by biofilm-forming bacterias. $^{4}$

There are two classical approaches for the treatment of infected non-union conventional and active approach. Conventional approach focuses on infection control first prior to bony union whereas active approach prioritizes achievement of bony union in presence of infection. ${ }^{5,6}$ Conventional treatment of non-union follows a two-stage procedure. In first stage wound is debrided, infected 
implant and necrotic tissues are removed and local (in the form of antibiotic cement beads) or systemic antibiotic is delivered to render it a aseptic non-union. Then in second stage stability is achieved by means of external or internal fixation. ${ }^{4}$ Single-staged procedures such as debridement and application of Ilizarov fixator or use of antibiotic cement-impregnated intramedullary nails (ACIINs) have been described in the literature. ${ }^{7}$

Ilizarov fixator is a revolutionary system when the bone deficit is more as it has the advantage of tissue regeneration in addition to external fixation. But problems with Ilizarov are pin tract infection, poor patient acceptability and long learning curve for the surgeons. ${ }^{8}$ Paley and Herzenberg first described the use of ACIIN. Major advantages of ACIIN are high local concentration antibiotics and low systemic side effects. Gentamycin and Vancomycin are most widely used antibiotics due to their broad spectrum activity and heat stability. ${ }^{9,10}$ With the advantage of being single staged procedure for infection control and fracture stability, cost effectiveness of implant we intended to study the outcome of ACIIN use in infected non-union of tibia and femur.

\section{METHODS}

This is a hospital based prospective case series type of descriptive study conducted in Department of Orthopedics, SMS Medical College and Hospital, Jaipur. Data collection for this study was done from Febraury 2017 to October 2018. We studied 35 cases of infected non-union of femur and tibia fracture with interlock nail in situ and minimum duration of 3 months with either of discharging sinus or swelling and tenderness at fracture site or laboratory parameters suggestive of presence of infection. Patients allergic to Gentamicin or Vancomycin or with bone defect $>3 \mathrm{~cm}$ or with raw area are excluded from the study.

Pre-operative evaluation includes complete blood count, erythrocyte sedimentation rate (ESR), C-reactive protein (CRP), standard radiograph. After pre-operative evaluation informed consent for surgery and participation in the study was taken. Nail size was measured. For tibia pre-operative measurement of length of nail was taken from tibial tubersity to the medial malleolus (TMD method) and for femur it was from tip of greater trochanter to upper pole of patella. ${ }^{11,12}$ Next step involved removal of interlocking nail and thorough debridement of the infected bone and soft tissues and then copious lavage. All the nonviable and infected soft tissues and bone were removed until bleeding viable tissue was present at the resection margins. Specimens were obtained of the bone, soft tissues, and any purulent material present and were sent for aerobic and anaerobic cultures and sensitivity. Adequate reaming of the intramedullary canal was done to accommodate larger size nail to have better stability. Antibiotic cement impregnated nail was prepared under sterile conditions in the operating room. The nail was prepared on a separate sterile table. V-Nail (for tibia) or Kuntscher nail /KNail(for femur ) of $8 \mathrm{~mm}$ or $9 \mathrm{~mm}$ diameter respectively was chosen and standard viscosity gentamicin bone cement were used. $40 \mathrm{gm}$ cement was thoroughly mixed with $2 \mathrm{gm}$ of vancomycin following which the monomer (liquid) was added. When the cement reaches doughy consistency, K-Nail/V-Nail was coated upto $1 \mathrm{~mm}$ less than the diameter of the last reamer and manually rolled to achieve uniform diameter. Proximal eye of nail left open while distal nail tip cement was moulded to smooth bullet-nose shape. The diameter required is checked with Kuntscher intramedullary nail gauge and excess cement was shaved off and nail rerolled before the cement set and diameter was rechecked. Bone cement was allowed to set for 15 minutes before insertion for the monomer to evaporate and to prevent cement nail debonding. Bone ends were aligned and nail was placed. Wound wash was done and wound closed. Above knee slab was applied for tibia and below knee slab with derotation boot in case of femur (Figure 1 and 2).

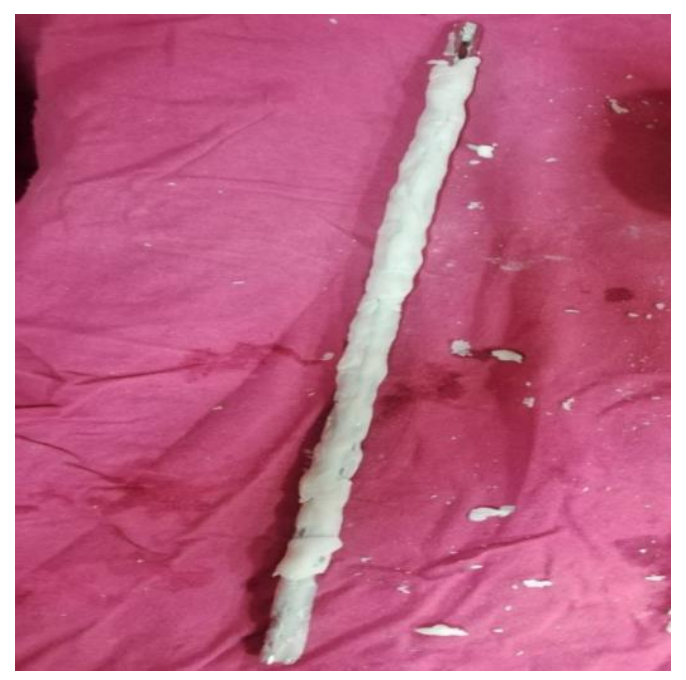

Figure 1: Antibiotic cement impregnated K-nail.

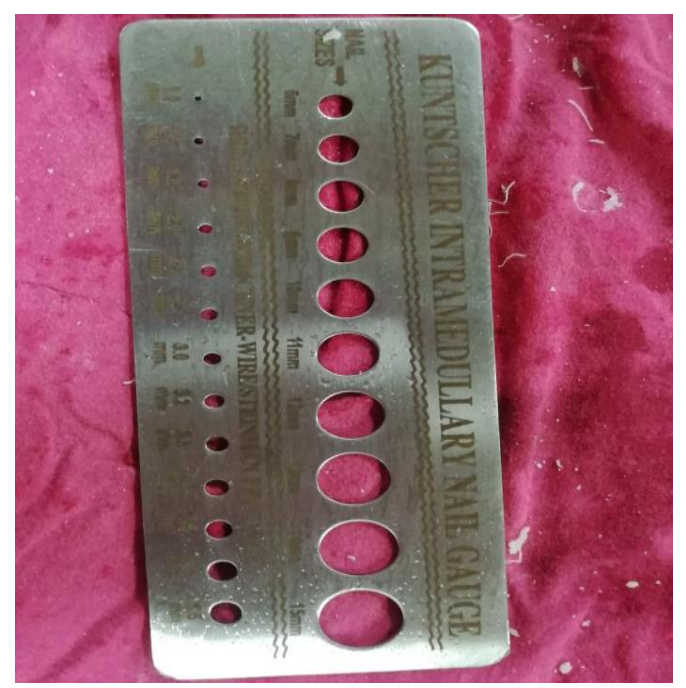

Figure 2: Kuntscher intramedullary nail gauge. 
Wound inspected at intervals of 48-72 hours. Ceftriaxone was administered during the first 24 to 48 hours after surgery until the results of culture and sensitivity from the samples sent at the time of surgery were available. The systemic antibiotics were then changed, if necessary, based on culture results. Further treatment with oral antibiotics continued for 6 weeks. Partial weight bearing with support was started at 6 weeks of fixation. A patellar tendon-bearing cast was applied for tibia and a hip-kneeankle- foot orthosis (HKAFO) for femur. Gradual full weight bearing was permitted. The cast continued till union. Clinical and radiological features were used to assess the progress of bony union at 2 week, 6 week, 3 month, 6 month, 9 month and 1 year or till union was sound. Active physiotherapy for regaining ankle and knee mobility were instituted till the range of movement was satisfactory. A complete blood count (CBC), erythrocyte sedimentation rate (ESR) and C-reactive protein (CRP) levels were performed pre-operatively and then at 2 week, 6 week, 3 month, 6 month, 9 month and 1 year to record rising or falling trends. In patients with limb shortening, heel and sole rise was given. The final results were evaluated by Paley's bony criteria and functional criteria. Data was analysed using SPSS 2013.

\section{RESULTS}

In our study among 35 patients 33 (94.29\%) were male and $2(5.71 \%)$ were female. Mean age of the patients was 37.3 years (range 16-64 years). Femur was affected in $62.86 \%$ cases and tibia in $37.14 \%$ cases. $68.57 \%$ cases in present study were initially compound and $32.43 \%$ cases were closed. Mean duration of follow up was 9.2 month. Infection was controlled in $94.28 \%$ cases (Table 1). Bony union was achieved in $88.57 \%$ cases (19 femur and 12 tibia). Average duration for bony union was 7.3 month for femur and 8 month for tibia. $54.55 \%$ femur united at 6 month, $27.27 \%$ femur united at 9 month, $4.55 \%$ femur united at 1 year and $13.64 \%$ femur did not unite. In case of tibia, $38.46 \%$ united at 6 month, $46.15 \%$ tibia united at 9 month, $7.69 \%$ united at 1 year and $7.69 \%$ cases did not unite (Table 2).

Table 1: Infection control.

\begin{tabular}{|lll|}
\hline No of cases & Infection controlled & Infection not controlled \\
\hline Percentage & 33 & 2 \\
\hline
\end{tabular}

Table 2: Distribution of cases according to Bony union.

\begin{tabular}{|ll|llllll|}
\hline & $\mathbf{6}$ months & $\mathbf{9}$ months & $\mathbf{1 2}$ months & Total united & Non united & Total \\
\hline Femur (N) & 12 & 6 & 1 & 19 & 3 & 22 \\
\hline Percentage (\%) & 54.55 & 27.27 & 4.55 & 86.36 & 13.64 & 100.00 \\
\hline Tibia (N) & 5 & 6 & 1 & 12 & 1 & 13 \\
\hline Percentage (\%) & 38.46 & 46.15 & 7.69 & 92.31 & 7.69 & 100.00 \\
\hline
\end{tabular}

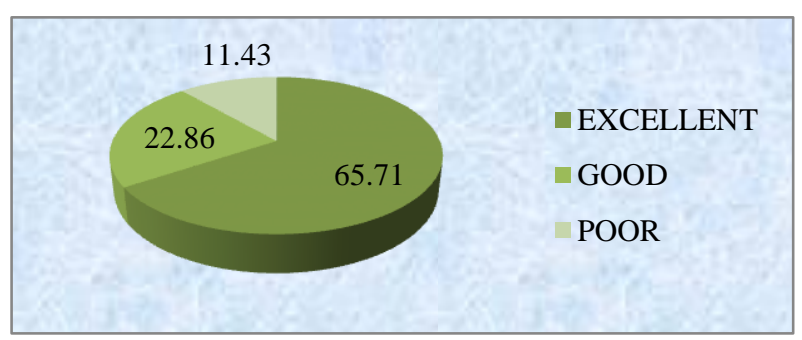

Figure 3: Distribution of cases according to Paley's criteria of bony outcome.

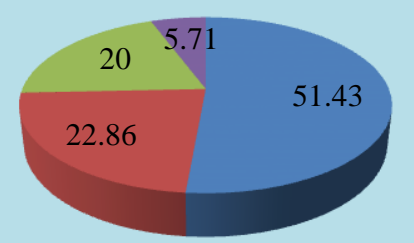

$\approx$ EXCELLENT $\backsim$ GOOD $\backsim$ FAIR $\backsim \mathrm{POOR}$

Figure 4: Distribution of cases according to Paley's functional outcome.

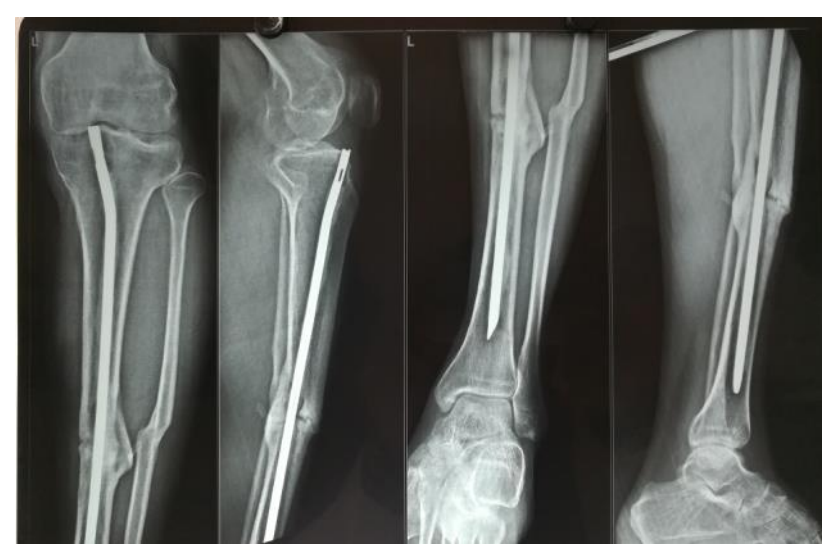

Figure 5: Radiograph of tibia treated by antibiotic coated V-nail at 9 months follow up.

According to PALEY'S criteria for bony outcome $65.71 \%$ had shown excellent outcome, $22.86 \%$ shown good outcome and $11.43 \%$ shown poor outcome. Complications encountered were difficulty in nail removal in two cases of $\mathrm{K}$-nail in femur and nail migration to ankle joint in one case and recurrence of 
infection in four cases. Five cases had undergone nail removal due to infection recurrence or nail migration, subsequent sequestrectomy was performed in $2.86 \%$ cases. Average limb length discrepancy was $1.2 \mathrm{~cm}$. As per PALEY'S criteria for functional outcome $51.43 \%$ patient had shown excellent, $22.86 \%$ shown good, $20 \%$ shown fair and $5.71 \%$ shown poor outcome (Figure 3 and 4) (Figure 5 and 6).

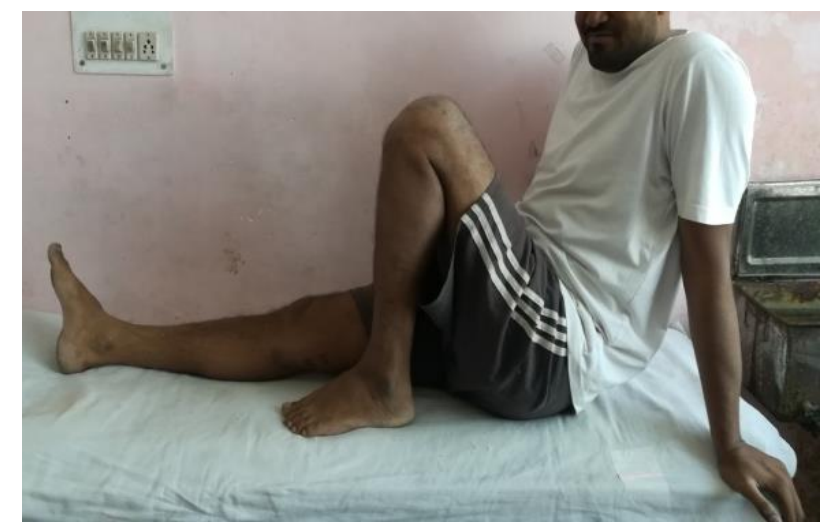

Figure 6: Clinical picture of patient of infected nonunion tibia at 9 months follow up with full knee flexion.

\section{DISCUSSION}

Treatment of infected nonunion of long bones is a big challenge. $^{3}$ There is no single universally accepted modality of treatment available for the management of this problem. Traditionally, infected non unions have been managed using two-step procedure to control the infection first and subsequently to treat the nonunion. Antibiotic cement impregnated intramedullary nail (Knail/V-nail) serves both purpose simultaneously. Long term infection and repeated debridement create excessive fibrosis around the nonunion site and hinder antibiotic permeability to the desirable level. ${ }^{13}$ With the help of ACIIN we can attain high concentration of antibiotic locally without systemic side effects and can have gradual release of antibiotic for long duration (upto 36 weeks). ${ }^{14}$ Antibiotics used for this purpose should have broad spectrum of activity, heat resistance, low allergenicity and good elusion property. Gentamycin and vancomycin are common choices. Elution of antibiotics follows a biphasic pattern, with an initial rapid phase and a secondary slow phase. Elution is at its maximum during the 1st day, greatly declines on the 2nd day, and then, gradually decreases over time and stabilize between days 5 and 10 and continues upto 36 weeks. ${ }^{5,14,15}$

Ilizarov fixator is an excellent modality to treat infected nonunion. Although it has a cumbersome assembly and is technically demanding and also has a low acceptability among patients along with a high prevalence of pin-site infections, muscle contractures and joint stiffness but whenever there is bone gap it remains the modality of choice.
Control of infection in 33 out of 35 cases in our study is comparable with result of 19 out of 20 cases in a study conducted by Bhatia et al, 24 out of 25 cases by Saravanan et al, 17 out of 18 cases by Zhang Qiang et al. $^{14-16}$

In terms of bony union, the results vary in different studies. We used antibiotic cement impregnated intramedullary nail as a mode of definitive fixation in contrast to Shyam et al who kept ACIIN for 6-12 weeks and subsequently depending upon bone gap he used exchange interlocking nail or Ilizarov methodology. Bony union was achieved in $88 \%$ cases (19 femur and 12 tibia). ${ }^{3}$ Conway et al reported bony union in $73 \%$ of the cases with antibiotic nail as the index procedure. ${ }^{17}$ Bhatia et al found bony union in $60 \%$ cases without secondary procedure. ${ }^{14}$ Paley et al have achieved bony union in about $80 \%$ cases by Ilizarov methodology in infected non-union of long bones. ${ }^{8}$

Average duration for bony union was 7.3 months for femur and 8 months for tibia in present study. Similarly average time taken for union in patients in whom antibiotic cement nailing was the only procedure required was about 32 weeks for tibia as shown by Bhatia et al. ${ }^{14}$ Time required for bony union of average 26 weeks for tibia and 24 weeks for femur by Saravanan et al and of 26.4 weeks for tibia and 31.5 weeks for femur by Han et al are also comparable with our results. ${ }^{15,18}$ One patient of femur in our study may unit on subsequent follow up as average time for union of femur in our study is 7.3 months and we could follow up this case upto 6 months only. Two patients those who did not show infection control and bony union could be due to their poor nutritional status, smoking habit and infection by organisms that are resistant to gantamycin and vancomycin although we did not study the reasons specifically.

Here we would like to draw the attention of readers that none of the patients who achieved bony union in our study required bone grafting as a secondary procedure whereas in previous studies researchers used bone grafting to achieve bony union in few cases.

Complications in our study were difficulty in nail removal in two cases of $\mathrm{K}$-nail in femur and nail migration to ankle joint in one case. Similar type of problems was encountered by other researchers also. Difficulty in nail removal is due to anchoring of nail in the canal by fibrosis and cement unbinding. It may also be attributed to faulty nail preparation in the form of inability to make a smooth surface. Recurrence of infection was a major issue which lead to implant removal which was another difficult job in some cases.

From this study we can come to the conclusion that antibiotic cement impregnated intramedullary nail is an effective means of controlling infection in infected non- 
union of long bones as well as good tool for achieving bony union.

Funding: No funding sources

Conflict of interest: None declared

Ethical approval: The study was approved by the institutional ethics committee

\section{REFERENCES}

1. Makridis KG, Tosounidis T, Giannoudis PV. Management of Infection After Intramedullary Nailing of Long Bone Fractures: Treatment Protocols and Outcomes. Open Orthopaed J. 2013;7(Suppl 2):219-26.

2. Nandakumar R, Mahapatra S. Infected non union of long bones- Is low cost antibiotic nail a viable option in Indian scenario. Int J Contemp Med Res. 2016;3(5):1492-5.

3. Shyam AK, Sancheti PK, Patel SK, Rocha S, Pradhan C, Patil A. Use of antibiotic cementimpregnated intramedullary nail in treatment of infected non-union of long bone. Indian J Orthop. 2009;43(4):396-402.

4. Stoodley P, Ehrlich GD, Sedghizadeh PP, Stoodley LH, Baratz ME, Altman DT, et al. Orthopaedic biofilm infections. Curr Orthop Pract. 2011;22(6):558-63.

5. McMurtry J, Mounasamy V. Antibiotic Intramedullary Nail in the Management of Infected Ununited Tibial Fractures: Case Report, Technique and Review of Literature. HSOA Journal of Clinical Studies and Medical Case Reports. 2016;3:029.

6. Chen CE, Ko JY, Wang JW, Wang CJ. Infection after intramedullary nailing of the femur. J Trauma. 2003;55(2):338-44.

7. Bar-On E, Weigl DM, Bor N, Becker T, Katz K, Mercado E, et al. Chronic osteomyelitis in children: treatment by intramedullary reaming and antibioticimpregnated cement rods. J Pediatr Orthop. 2010;30(5):508-13.

8. Paley D. Problems, obstacles, and complications of limb lengthening by the Ilizarov technique. Clin Orthop Relat Res. 1990;250:81-104.

9. Paley D, Herzenberg JE. Intramedullary infections treated with antibiotic cement rods: preliminary results in nine cases. J Orthop Trauma. 2002;16(10):723-9.

10. Dhanasekhar R, Jacob PJ, Francis J. Antibiotic cement impregnated nailing in the management of infected non-union of femur and tibia. Kerala $\mathbf{J}$ Orthopaed. 2013;26:93-7.

11. Venkateswaran B, Warner RM, Hunt N, Shaw DL, Tulwa N, Deacon P. An easy and accurate preoperative method for determining tibial nail lengths. Injury. 2003;34(10):752-5.

12. Lakhey S, Pradhan RL, Bishwakarma M, Pradhan S, Pradhanaga S, Pandey BK, et al. Pre-operative assessment of K-nail length in fracture shaft of femur. Kathmandu Univ Med J (KUMJ). 2006;4(3):316-8.

13. Rizk AS. Partial fibulectomy for treatment of tibial nonunion. Egyptian Orthop J. 2014;49:18-23.

14. Bhatia C, Tiwari AK, Sharma SB, Thalanki S, Rai A. Role of Antibiotic Cement Coated Nailing in Infected Nonunion of Tibia. Malaysian Orthop J. 2017;11:1.

15. Saravanan A, Ganesh RR, Deen N, Ismail M, Anandan H. Antibiotic cement impregnated nailing in management of infected non union of femur and tibia; Int J Sci Study. 2017;5:4.

16. Qiang Z, Jun PZ, Jie XJ, Hang L, Bing LJ, Cai LF. Use of antibiotic cement rod to treat intramedullary infection after nailing: preliminary study in 19 patients. Arch Orthop Trauma Surg. 2007;127(10):945-51.

17. Thonse R, Conway J. Antibiotic cement-coated interlocking nail for the treatment of infected nonunions and segmental bone defects. J Orthop Trauma. 2007;21(4):258-68.

18. Han SK, Choi NY, Park SJ, Lee SK, Jang G, Lee IJ, et al. Evaluate the results of treatment of antibiotic cement coated un reamed nailing for infected nonunion of long bones. J Korean Orthop Assoc. 2000;35(5):699-703.

Cite this article as: Sen A, Kulkarni C, Meena A. Descriptive study of outcome of antibiotic cementimpregnated intramedullary nail in treatment of infected non-union of weight bearing long bones. Int $\mathbf{J}$ Res Orthop 2019;5:1102-6. 